\title{
Kriging of Airborne Gravity Data in the Coastal Areas of the Gulf of Mexico
}

\author{
Hongzhi Song, Alexey L. Sadovski, Gary Jeffress \\ Conrad Blucher Institute, Texas A\&M University-Corpus Christi, Corpus Christi, USA \\ Email: Alexey.sadovski@tamucc.edu
}

Received July 2013

\begin{abstract}
This paper deals with the application of kriging technique to find the continuous map of gravity on the geoid in the coastal areas and to evaluate its precision.
\end{abstract}

Keywords: Gravity; Kriging; Geoid; Map; Statistics

\section{Introduction}

By using satellites, scientists discovered the long wave (large scale) geoid for the Earth (Seeber, 2003; Drinkwater et al., 2003) [1], but its resolution is not sufficient for orthometric height determination from GPS when it comes to the relatively small scale and/or local events such as flooding. This was the case after flooding created by storm surges from hurricanes Katrina, Rita (2005), and Ike (2008) in the coastal areas of the Gulf of Mexico. So, there is a need to develop method(s) and model(s) of the geoid determination at the local level, based on local observations of gravity, and complemented by observations of gravity from the air and space.

In principle, there is a need for gravity $g$ at every point of the Earth's surface. Gravity is continuously changing, and it reflects the results of Earth's phenomena, such as tropic storm, hurricane, earthquake, early tides, variation in the atmosphere density, etc. Gravity also alters when only a small change happened in the constructions and the density of materials beneath the constructions. But having gravity data provided everywhere on the Earth is totally impossible in reality. To predict values of a random unsampled area from a set of observations is needed. It is well known that the kriging method is not the best approach to predict free-air gravity anomalies, but in this paper, we assume that the kriging method is a better approach than other methods for prediction of gravity based on the airborne data provided by National Geodetic Survey (NGS). The result we still have a confidence in the kriging method is that the kriging method can estimate the prediction error to assess the quality of a prediction. This function makes the kriging method with a big difference from other methods.

\section{Data}

Data used in this chapter is airborne gravity data of the Gravity for the Redefinition of the American Vertical Datum (GRAV-D) project which was released by NGS [2]. Table 1 lists the nominal block characteristics, and details can be founded in GRAV-D General Airborne Gravity Data User Manual. Four blocks (Block CS01, CS02, CS03 and CS04) data (Figure 1) were chosen to be interpolated.

The total sample size (four blocks together) is 389578 , and the gravity values range between $975480 \mathrm{mGal}$ and $977490 \mathrm{mGal}$. Keep in mind, the standard gravity is $980665 \mathrm{mGal}$. The airborne gravity data was fixed by using free-air reduction and by the international gravity formula [3].

\section{Kriging of Gravity on the Geoid}

The kriging method here was conducted by using ArcGIS 10.1-Spatial Analyst and Geostatistical Analyst. There are six different types of kriging in Geostatistical Analyst tools. The most common types are ordinary kriging and universal kriging, which were chosen to be used in this study. The simple kriging method is also

Table 1. Nominal data characteristics.

\begin{tabular}{c|c|}
\hline Characteristic & Nominal Value \\
\hline Altitude & $20,000 \mathrm{ft}(\sim 6.3 \mathrm{~km})$ \\
\hline Ground speed & $250 \mathrm{knots}(250$ nautical miles $/ \mathrm{hr})$ \\
\hline Along-track gravimeter sampling & 1 sample per second $=128.6 \mathrm{~m}$ (at nominal ground speed) \\
\hline Data Line Spacing & $10 \mathrm{~km}$ \\
\hline Data Line length & $400 \mathrm{~km}$ \\
\hline Cross Line Spacing & $40-80 \mathrm{~km}$ \\
\hline Cross Line Length & $500 \mathrm{~km}$ \\
\hline Data Minimum Resolution & $20 \mathrm{~km}$ \\
\hline
\end{tabular}




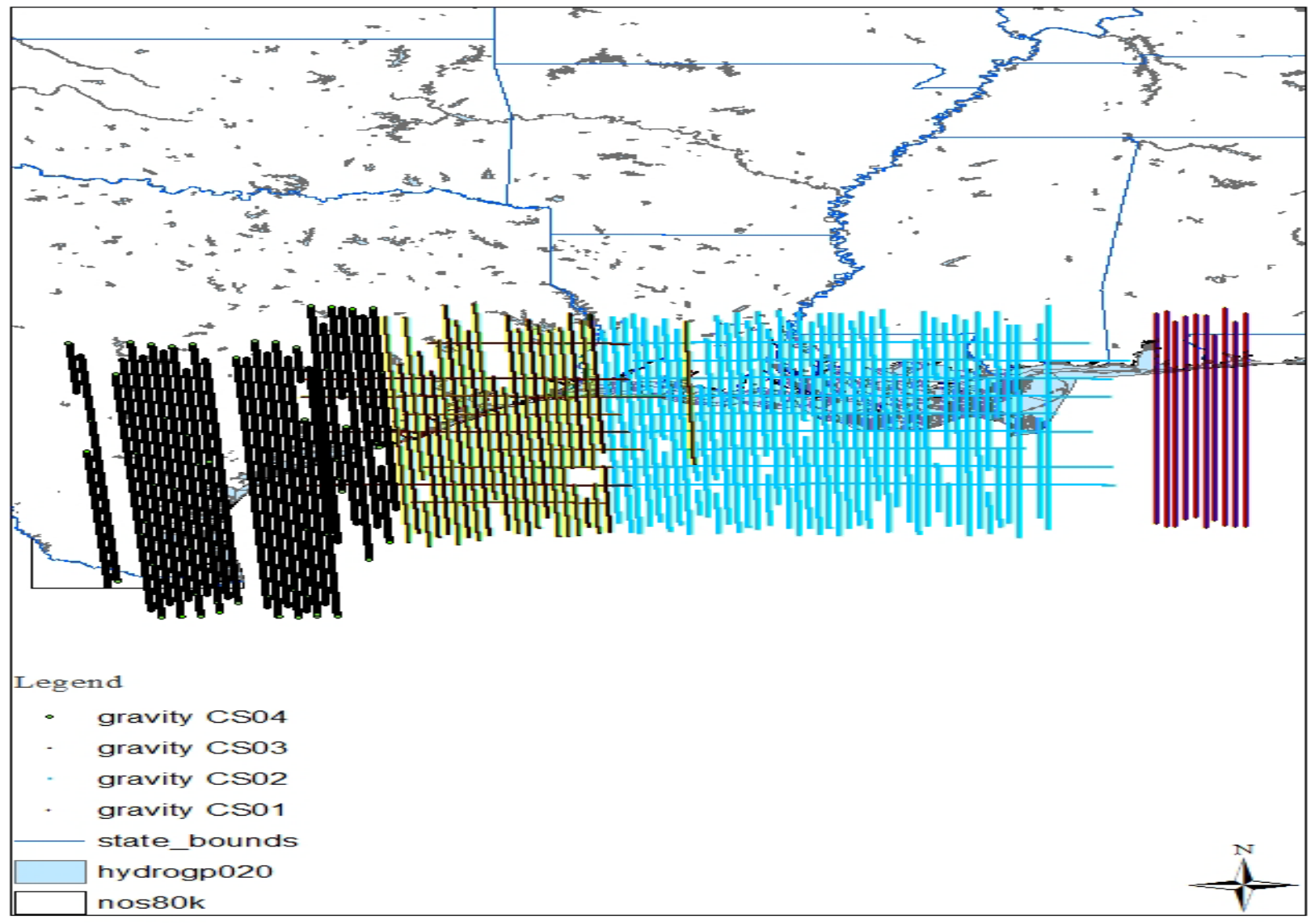

Figure 1. Tracks and locations of data of airborne gravity.

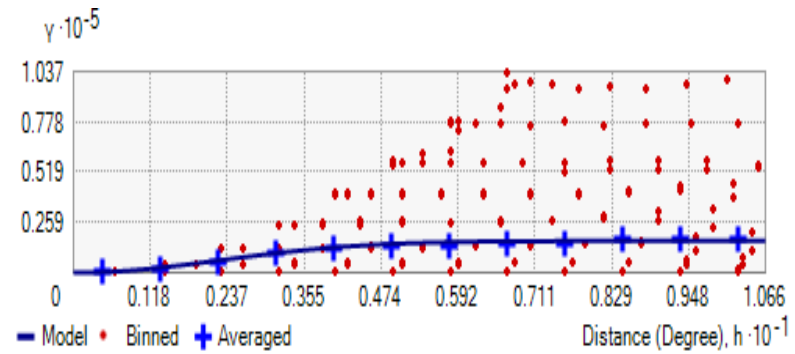

Figure 2. The average semivariogram values.

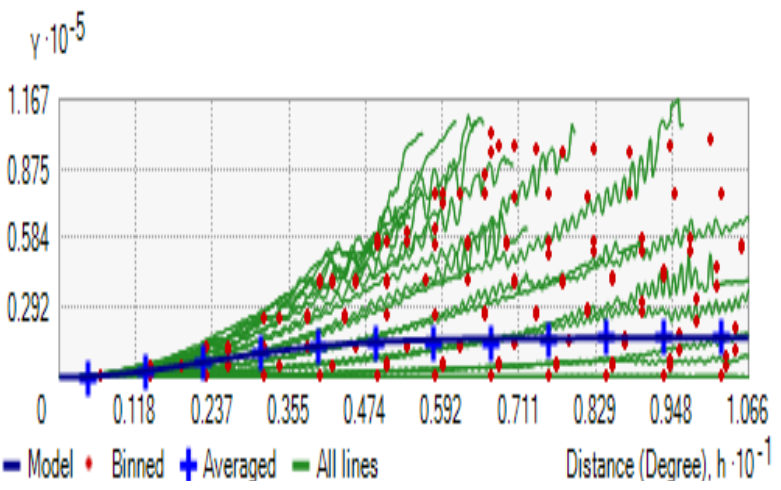

Figure 3. Semivariogram with all lines (green lines) which fit binned semivariogram values.

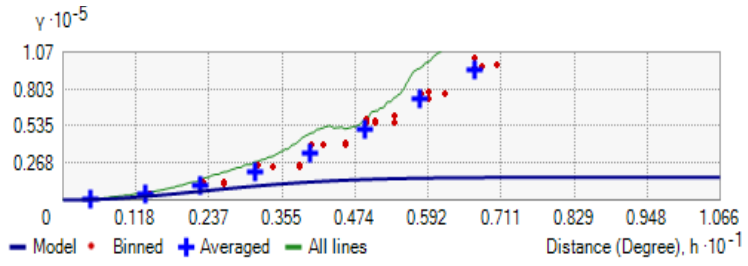

Figure 4. Semivariogram with showing search direction. The tolerance is $\mathbf{4 5}$ and the bandwidth (lags) is 3 . The local polynomial shown as a green line fits the semivariogram surface in this case.

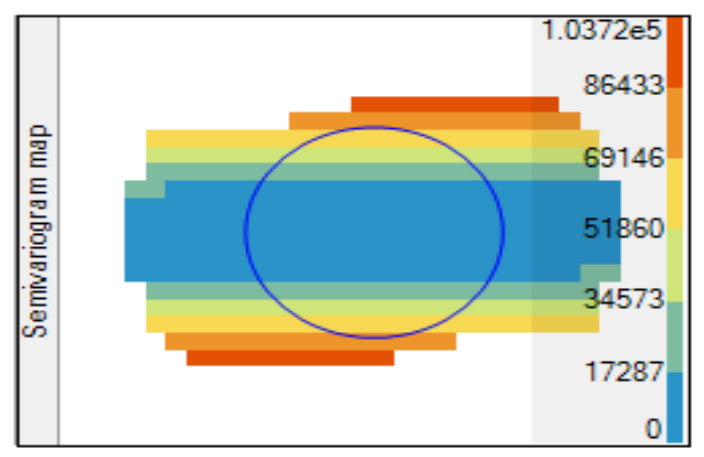

Figure 5. A semivariogram map. The color band shows semivariogram values with weights. 
quite common, but it requires that the data should have normal distribution. Thus, the simple kriging method was not applied in this study. There are three major components - the spatial autocorrelation component (known as semivariogram), a trend, and a random error term. These three components are the key to lead to different types of the kriging methods. The simple equation represents the kriging method is: $z_{s}=\sum_{i=1}^{n} z_{i} w_{i}$, where $z_{s}$ is the estimated value for an unsampled location $s ; z_{i}$ is the known value at the control point $i ; w_{i}$ represents the weight applied to sample values associated with the control point $i$; $\mathrm{n}$ is the number of sample points used in the estimation. The averaged semivariogram values on the $y$-axis (in $\mathrm{mgal}^{2}$ ), and distance (or lag) on the $\mathrm{x}$-axis (in degree). Binned values are shown as red dots, which are sorted the relative values between points based on their distances and directions and computed a value by square of the difference between the original values of points; Average values are shown as blue crosses, which are generated by binning semivariogram points; The model is shown as blue curve, which is fitted to average values. Model: $28.118 *$ Nugget $+16437 *$ Stable $(5.53,2)$; Model: $28.118 *$ Nugget $+16437 *$ Gaussian (5.53).

The predicted, error, standard error, and normal QQ plot graphs are plotted respectively in Figures 6(a)-(d). The predicted graph shows how well the known sample

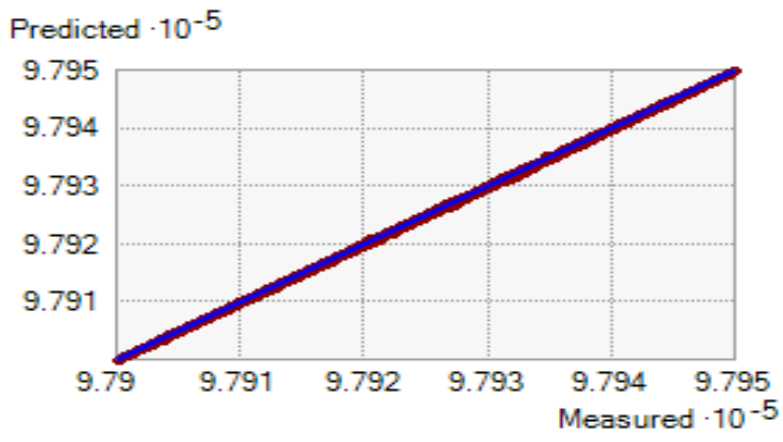

(a)

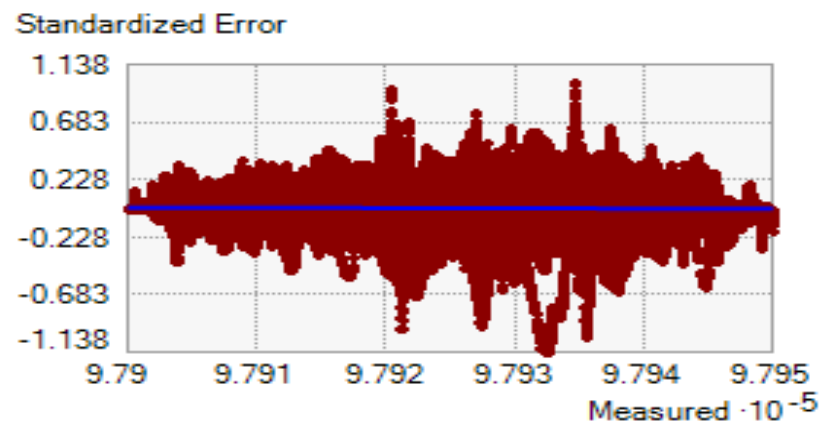

(c) value was predicted compared to its actual value. The regression function in Figure 6(a) is

$f(x)=0.9999 x+125.1751$. By visually analyzed the graph, the regression function is closely aligned with the reference line. Therefore, it is well predicted.

The error graph shows the difference between known values and predictions for these values. The error equation in Figure 6(b) is $y=10.00001 x+127.1751$. The standardized error graph shows the error divided by the estimated kriging errors. The standardized error equation in Figure 6(c) is $y=10.00002 x+22.9974$. The normal QQ plot of the standardized error Figure 6(d) shows how closely the difference between the errors of predicted and actual values align with the standard normal distribution (the reference line). Figures 7 and 8 demonstrate the prediction and standard error map by using the ordinary kriging with stable and Gaussian techniques.

Trend analysis was presented in Figure 9. There is no trend exists because the curve through the projected points is flat (as shown by the light blue line in the Figure 9). A slight downward curve as shown by the red line in Figure 9 is through the projected points on $\mathrm{ZY}$ plane, which suggests that it may have a trend exist in gravity on the geoid data. Therefore, de-trend is conducted before the universal kriging process in order to prevent biased the analysis. Because the curve shown on ZY plane is not obvious, the de-trend approach is chosen

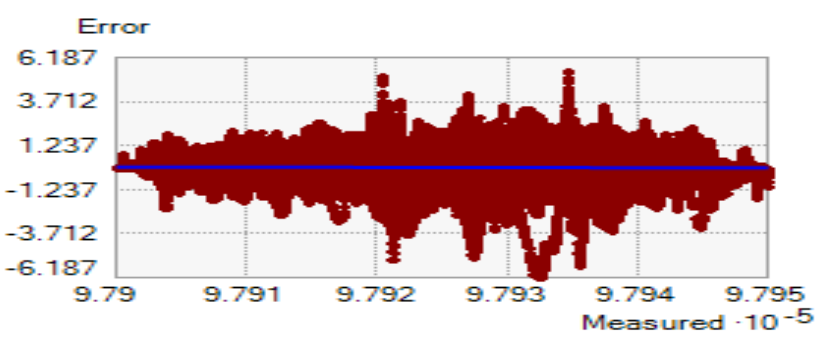

(b)

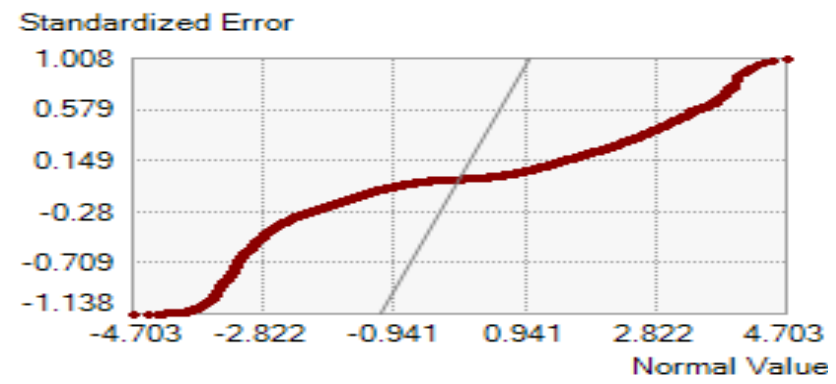

(d)

Figure 6. Cross validation of the ordinary kriging. 


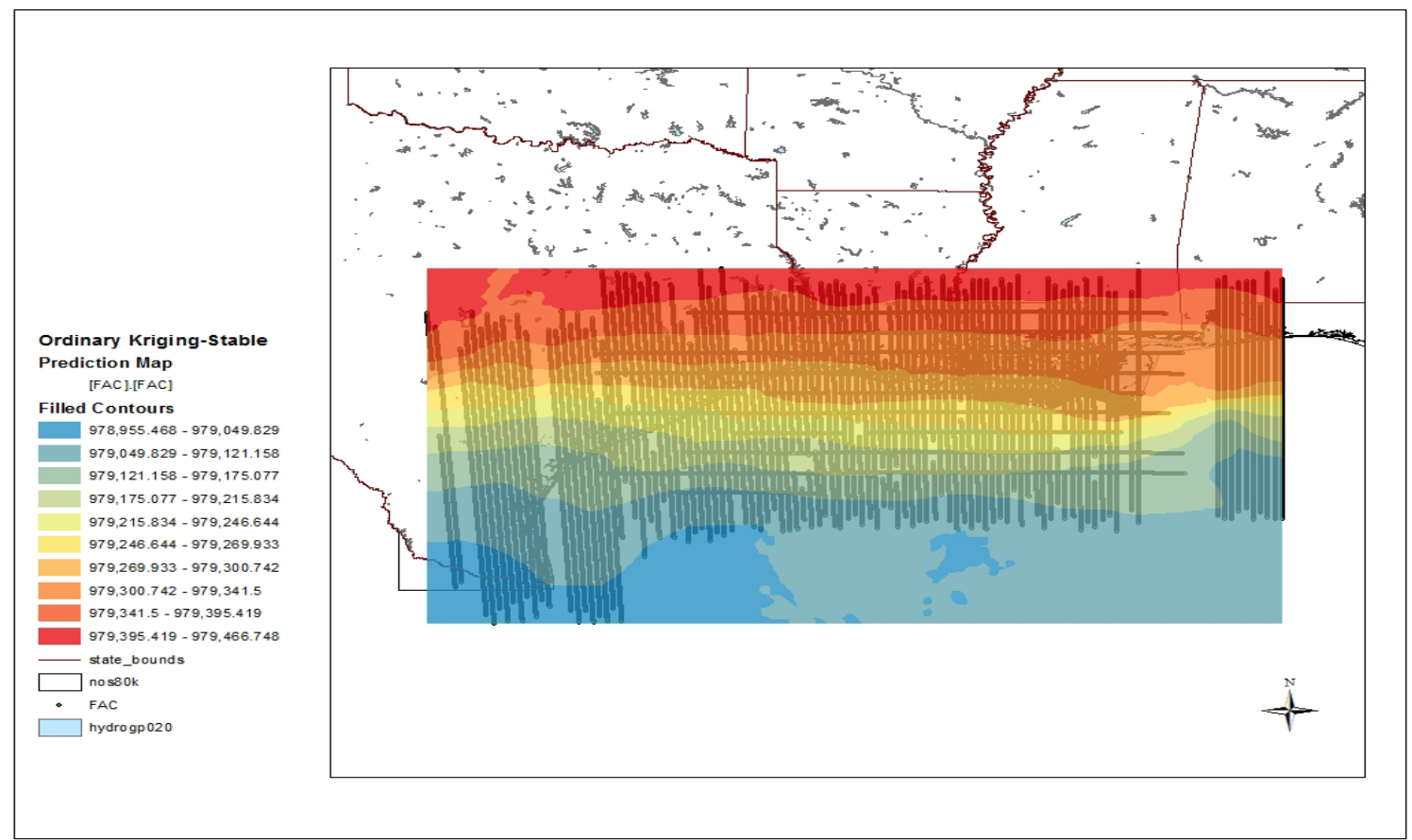

Figure 7. The ordinary stable and Gaussian kriging predictions map.

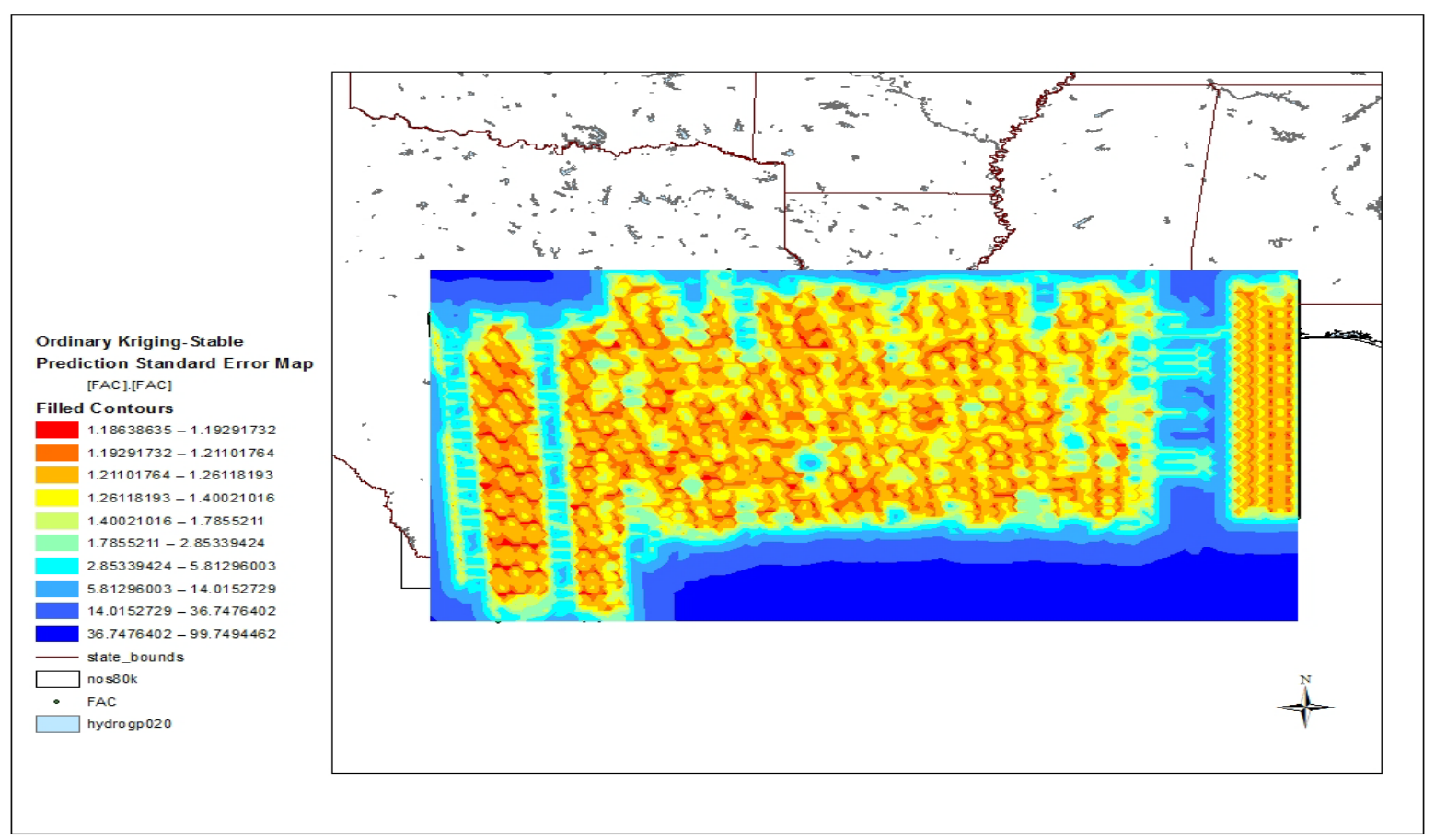

Figure 8. The ordinary stable and Gaussian kriging prediction standard error map.

to remove the trend order as constant. The process was conducted in ArcGIS 10.1 by using Geostatistical Analyst. Results of the universal kriging with either the stable technique or the Gaussian technique were shown as exact the same as results of the ordinary kriging.

Legend: Grid (XYZ): Number of Grid Lines $11 \times 11 \times$
6; Projected Data: YZ plane (Dark Blue), ZY plane (Yellow), XY plane (Peony Pink); Trend on Projections: YZ plane (Light Blue), XZ plane (Red); Axes (Black).

A better interpolation method should have a smaller RMS. Due to no difference between the ordinary kriging and universal kriging in this case; statistical results were 
Table 2. Statistics.

\begin{tabular}{cc}
\hline RMS Standardized & 0.1084 \\
\hline Mean Standardized & 0.0007 \\
Average Standard Error (ASE) & 5.5060 \\
Root Mena Square (RMS) & 0.5918 \\
Difference between & 4.9142 \\
RMS and ASE & $89.25 \%$ \\
\hline
\end{tabular}

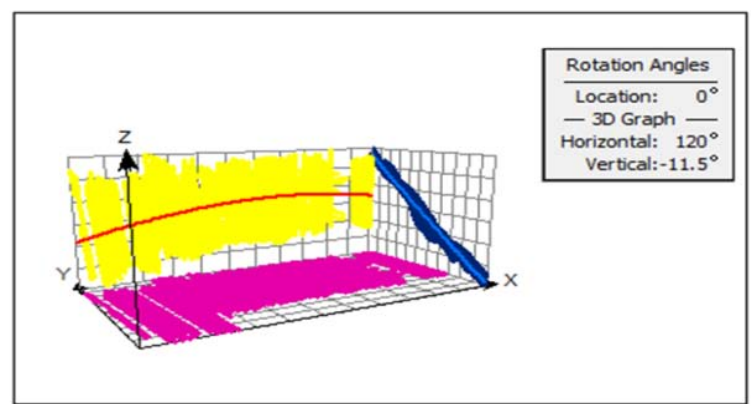

Figure 9. Trend analysis of gravity on the geoid.

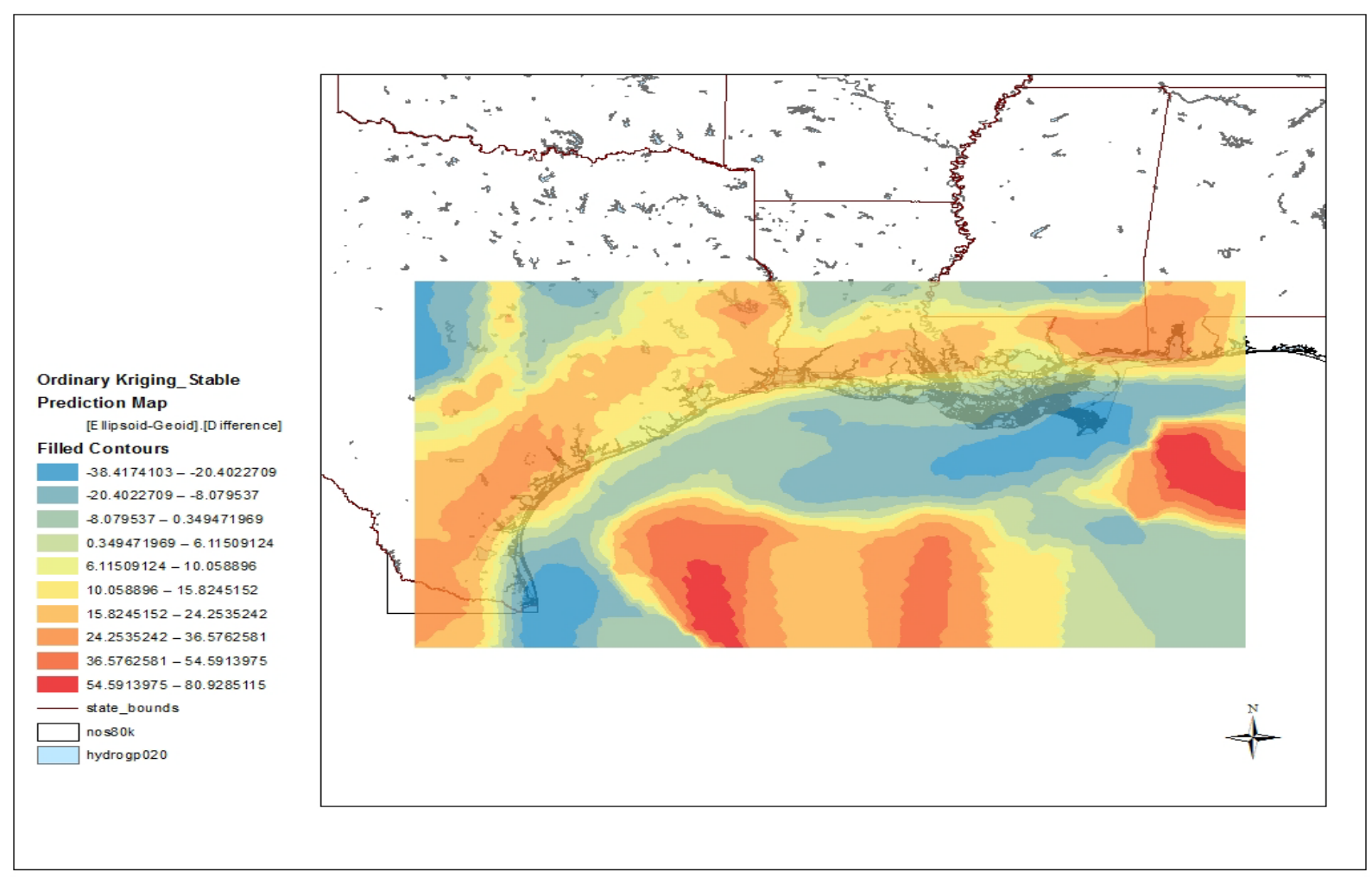

Figure 10. The ordinary kriging predictions map of gravity on the geoid along Gulf of Mexico coast.

the same which listed in Table 2. The prediction error mean is $0.0038 \mathrm{mGal}$. As 1 meter increased in altitude, the gravity is decreased by $0.3086 \mathrm{mGal}$. With simple conversion, the accuracy of prediction is approximately 0.0123 meters. Namely, it is around $1.23 \mathrm{~cm}$, which was expected.

\section{REFERENCES}

[1] M. R. Drinkwater, R. Floberghagen, R. Haagmans, D. Muzi and A. Popescu, "VII: CLOSING SESSION: GOCE: ESA's First Earth Explorer Core Mission,” Space Science Reviews, Vol. 108, No. 1-2, 2003, pp. 419-432.
http://dx.doi.org/10.1023/A:1026104216284

[2] GRAV-D Science Team, "GRAV-D General Airborne Gravity Data User Manual,” Theresa M. Diehl, Ed., Version 1, 2011.

http://www.ngs.noaa.gov/GRAVD/data_cs01.shtml

[3] X. Li and H. J. Götze, "Tutorial Ellipsoid, Geoid, Gravity, Geodesy, and Geophysics,” Geophysics, Vol. 66, No. 6, 2001, pp. 1660-1668. http://dx.doi.org/10.1190/1.1487109

[4] B. Hofmann-Wellenhof and H. Moritz, "Physical Geodesy,” 2nd Edition, Springer Wien, New York, 2006. 